\title{
Diffusion of colloidal particles in a tilted periodic potential: Theory versus experiment
}

\author{
Mykhaylo Evstigneev, ${ }^{1, *}$ Olga Zvyagolskaya, ${ }^{2}$ Stefan Bleil, ${ }^{2}$ Ralf Eichhorn, ${ }^{1}$ Clemens Bechinger, ${ }^{2}$ and Peter Reimann ${ }^{1}$ \\ ${ }^{1}$ Fakultät für Physik, Universität Bielefeld, 33615 Bielefeld, Germany \\ ${ }^{2}$ Physikalisches Institut, Universität Stuttgart, 70550 Stuttgart, Germany
}

(Received 11 January 2008; published 8 April 2008)

\begin{abstract}
We investigate the diffusion of a colloidal particle in a tilted periodic potential created by means of ten rotating optical tweezers arranged on a circle. Because of the viscous drag, the trap rotation leads to the onset of a tilting force in the corotating reference frame, so that in that frame the system can be described as an overdamped Brownian particle in a tilted periodic potential. The excellent agreement of the velocity and diffusion coefficient as a function of rotating frequency with theoretical predictions allowed us to extract the main parameters characterizing the system - the coefficient of free thermal diffusion and the potential corrugation depth-from the experimental results.
\end{abstract}

DOI: 10.1103/PhysRevE.77.041107

PACS number(s): 05.40.- a, 05.60.-k, 82.70.Dd

\section{INTRODUCTION}

When a colloidal particle is placed in a laser beam, then the oscillating electric field $E(t)$ of the laser will induce a dipole moment $p(t) \propto E(t)$ in the particle, whose timeaveraged energy of interaction with the field will be proportional to the intensity of the laser, $-\langle p(t) E(t)\rangle \propto-I$. Therefore, the particle will be drawn into that region of space, where the intensity of the laser is the largest, i.e., the laser beam will act as a trap for the colloidal particle. Diffusion of colloidal particles in periodic structures created by such optical traps has two aspects interesting from the point of view of controlling the particles' motion and particle sorting.

First, the free thermal diffusion of an overdamped Brownian particle in thermal equilibrium (as described by Einstein [1]) is always reduced if an additional external potential is switched on [2]. In nonequilibrium, the opposite can be true. It has been shown theoretically [3-6] that the diffusion coefficient of a single Brownian particle in a tilted periodic potential can become arbitrarily large. This finding has been confirmed experimentally for the diffusion of a single colloidal particle in a potential created by periodically arranged optical traps $[7,8]$.

Second, it is well known that the presence of geometric confinement makes the diffusive motion of the particles anisotropic and reduces their diffusion coefficient [9]. So far, this effect has been studied for symmetric geometries, such as diffusion in the vicinity of a wall, between two parallel walls [10], or within a cylinder [11]. The experiments involve observing the free diffusion of the particle and do not allow one to control the particle motion. The study of particle diffusion in a laser-created periodic potential has the advantage that the direction of the particle's diffusive motion can be preset by the experimentalist; this ability may be crucial when one measures the components of the diffusion tensor in more complex geometries than the ones mentioned above.

In the present work, we study the diffusion of a single colloidal particle in a potential created by ten laser traps

\footnotetext{
*mykhaylo@physik.uni-bielefeld.de
}

arranged on a rotating circle. Due to the strong confinement of the particles in the laser traps, the radial fluctuations of the particle's position are negligible in comparison to the rotational motion along the circle. Therefore, by switching into the corotating reference frame, one can describe the particle's motion as one-dimensional diffusion in a tilted periodic potential generated by the laser traps, where the tilt arises due to the Stokesean viscous drag on the particle. Furthermore, while the particle's mass is of importance in some situations and may lead to some interesting peculiarities in the particle's motion (see, e.g., $[12,13]$ ), in our setup, inertia effects are so small that they can safely be neglected; in other words, the dynamics of the particle can be regarded as overdamped.

We focus on the velocity (in the corotating reference frame) and the diffusion coefficient of the particle as functions of rotation frequency; analytical expressions for both of these quantities for an overdamped Brownian dynamics in a tilted periodic potential are available $[3,4]$. While the velocity increases monotonically with the tilt (which is proportional to the rotation frequency), the diffusion coefficient is known to exhibit a peak at some tilt value; this phenomenon is known as giant diffusion [3,4]. Using the theoretical results of $[3,4]$ for a single-particle diffusion in a periodic potential, we determine the parameters of the laser potential and the coefficient of free thermal diffusion of a colloidal particle in our experiment.

\section{EXPERIMENTAL SETUP}

As a sample cell we used a thin glass cuvette which contained an aqueous suspension of negatively charged silica beads of radius $b=1.5 \mu \mathrm{m}$. Due to gravity, the negatively charged silica plates forming the sample cell and the incident laser beam, the particles are confined to a horizontal plane where they perform Brownian motion. Vertical fluctuations are reduced to less than $0.1 \mu \mathrm{m}$. The particles are imaged with an inverted microscope onto a charge-coupled device (CCD) camera and the trajectories are monitored by means of digital video microscopy.

Using scanning optical tweezers based on an acoustooptical deflection system (for details of the setup, see [14]), 
we created a circular rotating trap array consisting of ten individual laser spots regularly arranged on a circle of radius $R=5 \mu \mathrm{m}$. We rotated the trap array with different angular frequencies $\Omega$. Within the corotating reference frame, the rotation of the traps manifests itself as a tilting Stokes force on the particles in a periodic potential [15]. Experimentally we changed the potential tilt by varying the frequency of rotation $\Omega$. The possibility to simultaneously generate several independent circular trap arrays allowed us to study the particles' motion at different potential tilts within a single experimental run.

Experimentally, the velocity and diffusion coefficient have been determined as ensemble-averaged long-time limits

$$
v=\lim _{t \rightarrow \infty} \frac{\langle x(t)\rangle}{t}, \quad D=\lim _{t \rightarrow \infty} \frac{\left\langle[x(t)-\langle x(t)\rangle]^{2}\right\rangle}{2 t},
$$

where $x(t)$ represents the position of any particle on the circle in the corotating frame of reference [16]. These estimates converged satisfactorily well after about $10 \mathrm{~s}$.

\section{DIFFUSION OF A SINGLE PARTICLE AND DETERMINATION OF MODEL PARAMETERS}

To model the motion of the particle theoretically, we employ the one-dimensional Langevin equation

$$
\gamma \dot{\tilde{x}}(t)=-U^{\prime}(\tilde{x}+R \Omega t)+\sqrt{2 \gamma k_{\mathrm{B}} T} \xi(t)
$$

with $\xi(t)$ being Gaussian white noise of unit strength,

$$
\langle\xi(t)\rangle=0, \quad\left\langle\xi(t) \xi\left(t^{\prime}\right)\right\rangle=\delta\left(t-t^{\prime}\right) .
$$

Here, $\tilde{x}$ denotes the position of the particle along the circle of radius $R=5 \mu \mathrm{m}$, the periodic potential $U(\widetilde{x})$ is rotating with angular frequency $\Omega, \gamma$ is the coefficient of viscous friction, and $k_{\mathrm{B}} T$ is the thermal energy.

By means of the transformation of variables

$$
x=\tilde{x}+R \Omega t,
$$

we switch to the corotating reference frame, where the Langevin equation of motion has the form

$$
\gamma \dot{x}(t)=-U^{\prime}(x)+\gamma R \Omega+\sqrt{2 \gamma k_{\mathrm{B}} T} \xi(t) .
$$

This is the equation of motion of a Brownian particle in a periodic potential $U(x)$ tilted by a constant force $F=\gamma R \Omega$.

The quantities of main interest are the velocity $v$ and the diffusion coefficient $D$ in the corotating reference frame, defined exactly as in the experiment via Eq. (1) [16], as functions of the rotation frequency $\Omega$. Presented in Fig. 1 (symbols) are the experimental results, where these quantities in the rotating reference frame are shown for three different laser intensities.

At high rotation frequencies, the value of the tilt exceeds the force due to the periodic potential, so that the latter can be neglected in comparison to the former. It then follows from Eq. (5) that the velocity approaches the asymptotic value $v \propto R \Omega$ and the diffusion coefficient assumes the free value given by the Einstein relation (8) (see below) at high $\Omega$.
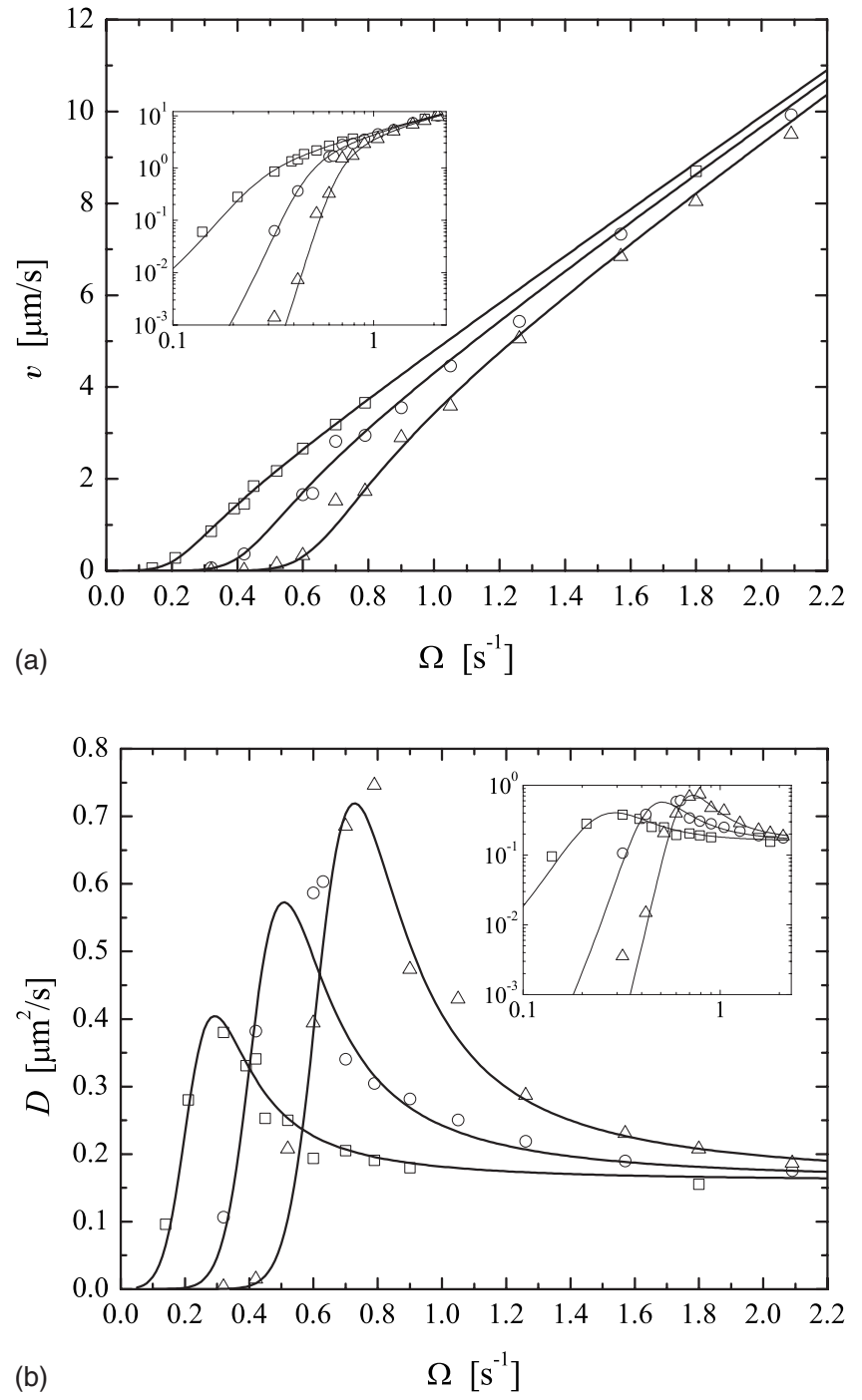

FIG. 1. Velocity and diffusion coefficient of a single colloidal particle of radius $b=1.5 \mu \mathrm{m}$ in the corotating reference frame [16] obtained experimentally according to Eq. (1) for ten laser traps rotating with angular frequency $\Omega$ on a circle with radius $R$ $=5 \mu \mathrm{m}$ (symbols). Squares, circles, and triangles correspond to three different laser intensities which are increasing but have not been calibrated quantitatively. Solid lines: corresponding theoretical predictions according to the exact analytical expressions (9)-(11) with the fit value of the free diffusion coefficient $D_{0}=0.16 \mu \mathrm{m}^{2} / \mathrm{s}$, and barrier heights $\Delta U_{0}=9 k_{\mathrm{B}} T$ (squares), $16 k_{\mathrm{B}} T$ (circles), and $23 k_{\mathrm{B}} T$ (triangles), and periodicity $L \simeq 3.14 \mu \mathrm{m}$. Insets show the same plots, but on the log-log scale.

On the other hand, at low rotation frequencies, the total potential in which the particle finds itself, $U(x)-\gamma R \Omega x$, consists of sequential potential wells separated by potential barriers. The motion of the particle in this regime is governed by the thermally activated hopping between the neighboring potential wells. The rate of this process is given by the Kramers law, $\omega_{\text {Kramers }} \propto e^{-\Delta V(\gamma \Omega R) / k_{\mathrm{B}} T}$, where $\Delta V(F)$ represents the height of the potential barrier that needs to be surmounted in order for the particle to move into an adjacent potential well. It is a decreasing function of the tilt, $F=\gamma \Omega R$. Correspondingly, both the velocity and the diffusion coefficient increase 
exponentially with the rotation frequency in this thermally activated regime. Interestingly, at the tilt close to the critical value, at which the barrier height of the potential $U(x)$ $-\gamma R \Omega x$ just vanishes, the diffusion coefficient exhibits a maximum.

Our goal is to determine the experimentally unknown properties of the system on the basis of the measured velocity and diffusion coefficient of the particle as a function of the rotation frequency; see Fig. 1. Once this is accomplished, a quantitative comparison of experimental and theoretical velocity and diffusion coefficient in Fig. 1 is straightforward.

The first such unknown system property is the periodic potential $U(x)=U(x+L)$. Recalling that in the experiment ten laser spots are regularly arranged on a circle with radius $R$ $=5 \mu \mathrm{m}$, the period of the potential follows as $L=2 \pi R / 10$ $\simeq 3.14 \mu \mathrm{m}$. Next, we analyzed the experimentally observed particle probability distributions for the different laser intensities and rotation frequencies $\Omega$ according to the method described in [8] with the result that in all cases the potential can be very well approximated by the simple functional form

$$
U(x)=-\frac{\Delta U}{2} \cos \frac{2 \pi x}{L}
$$

with unknown amplitude $\Delta U$.

Further experimentally unknown quantities of importance are the temperature $T$, which exceeds the ambient laboratory temperature by an unknown amount due to the heating effect of the laser, and the friction coefficient $\gamma$, which differs from the Stokes friction formula for a spherical particle in water at a given $T$ by an unknown amount due to the hydrodynamic interaction of the particle with the bottom of the glass cuvette.

The above three unknown parameters $\Delta U, \gamma$, and $T$ are not independent and cannot be determined from the experimental data uniquely. To see why this is so, we divide Eq. (5) by $k_{\mathrm{B}} T$ in order to bring it into a form containing, instead of these three unknowns, only two ratios thereof:

$$
\dot{x}(t) / D_{0}=-U^{\prime}(x) / k_{\mathrm{B}} T+R \Omega / D_{0}+\sqrt{2 / D_{0}} \xi(t),
$$

where the coefficient of free thermal diffusion $D_{0}$ satisfies the Einstein relation [1]

$$
D_{0}=k_{\mathrm{B}} T / \gamma .
$$

In other words, we are effectively left with two unknown system parameters, namely, $\Delta U /\left(k_{\mathrm{B}} T\right)$ and $D_{0}$, and our goal is to determine them from the experimentally observed velocities and diffusion coefficients in Fig. 1. Accordingly, we now turn to the description of our fitting procedure.

In a first step, we analyze the general functional form of the velocity and diffusion coefficient. Corresponding to the Langevin equation (5) is the Fokker-Planck equation for the probability density $P(x, t)$ to find the particle in an infinitesimal interval around the point $x$ at the moment of time $t$ [17]: $\frac{1}{D_{0}} \frac{\partial P}{\partial t}=\frac{\partial}{\partial x}\left[\frac{\partial P}{\partial x}+\left(\frac{U^{\prime}(x)}{k_{\mathrm{B}} T}-\frac{R \Omega}{D_{0}}\right) P\right]$. Furthermore, for our purposes [namely, the determination of velocity and diffusion coefficient according to (1)] it is sufficient to focus on initial conditions of the form $P(x, 0)=\delta(x)$. Then, the time dependence enters the solution $P(x, t)$ merely in the form of a product
$D_{0} t$, and the four parameters $\Delta U, k_{B} T, D_{0}$ [or $\gamma$, cf. (8)], and $\Omega$ enter only in the form of the two ratios $\Delta U / k_{\mathrm{B}} T$ and $\Omega / D_{0}$. Therefore, if the average value of some $x$-dependent function $A(x), \int d x A(x) P(x, t)=\langle A\rangle\left(D_{0} t ; \Delta U / k_{\mathrm{B}} T, \Omega / D_{0}\right)$, increases linearly in time-which is the case for $A(x)=x$ and $A(x)=(x-\langle x\rangle)^{2}$ - then the corresponding rate of increase [see Eq. (1)] has the following scaling form:

$$
v=D_{0} \Phi\left(\frac{\Delta U}{k_{\mathrm{B}} T}, \frac{\Omega}{D_{0}}\right), \quad D=D_{0} \Psi\left(\frac{\Delta U}{k_{\mathrm{B}} T}, \frac{\Omega}{D_{0}}\right)
$$

for the dependence of the velocity and diffusion coefficient, respectively, on the four parameters $\Delta U, k_{B} T, D_{0}$ (or $\gamma$ ), and $\Omega$

With respect to the specific model (5), analytic expressions for the scaling functions $\Phi$ and $\Psi$ are available. Adopting Eqs. (18), (21), and (22) of Ref. [4], we can express them in terms of the unknown fit parameters $u=\Delta U / k_{\mathrm{B}} T$ and $w$ $=\Omega / D_{0}$ as follows:

$$
\Phi(u, w)=L \frac{1-e^{-L R w}}{\int_{0}^{L} d x I_{+}(x ; u, w)},
$$

$$
\Psi(u, w)=L^{2} \frac{\int_{0}^{L} d x I_{+}^{2}(x ; u, w) I_{-}(x ; u, w)}{\left(\int_{0}^{L} d x I_{ \pm}(x ; u, w)\right)^{3}}
$$

with

$$
\begin{aligned}
I_{ \pm}(x ; u, w)= & \int_{0}^{L} d z \exp \left[\mp \frac{u}{2}\left(\cos \frac{2 \pi x}{L}-\cos \frac{2 \pi(x \mp z)}{L}\right)\right. \\
& -R w z] .
\end{aligned}
$$

The functional form (9) suggests that, when trying to deduce the parameter $\Delta U / k_{\mathrm{B}} T$ and $\Omega / D_{0}$ by fitting the experimental graphs $v$ vs $\Omega$ and $D$ vs $\Omega$, it is advantageous to use a $\log -\log$ scale. On this scale, changing the fit parameter $D_{0}$ leads to a uniform translation of the fitting curves in both $x$ and $y$ directions, leaving their shapes invariant. On the other hand, the shape of these curves is controlled by the fit parameter $\Delta U / k_{\mathrm{B}} T$. This observation implies that the fit parameters $\Delta U / k_{\mathrm{B}} T$ and $D_{0}$ can be estimated independently and suggests the following fitting procedure consisting of two steps.

(1) For an arbitrarily chosen free thermal diffusion coefficient $D_{0}$, adjust the ratio $\Delta U / k_{\mathrm{B}} T$ so that the fit curves $v$ vs $\Omega$ and $D$ vs $\Omega$ have the same shape on the $\log$ - $\log$ scale as the experimentally measured ones; this is achieved by visual inspection of the respective curves.

(2) Adjust $D_{0}$ so as to make the fitting curves coincide with the experimental ones.

We applied this procedure to the experimental data for the velocity and diffusion coefficient as functions of the rotation frequency, and further refined the obtained results by numerically minimizing the discrepancies between the theoretical 
and experimental curves, that is, by minimizing the quantities $\Sigma_{i}\left[v\left(\Omega_{i}\right)-v_{i}\right]^{2}$ and $\sum_{i}\left[D\left(\Omega_{i}\right)-D_{i}\right]^{2}$. Here, summation is over all rotation frequencies $\Omega_{i}$ probed experimentally, $v_{i}$ and $D_{i}$ are the corresponding experimental values of the velocity and the diffusion coefficient, and $v(\Omega)$ and $D(\Omega)$ are the theoretical values from Eq. (9). We have found that such a refinement leads to only small changes of the fit values. Figure 1 shows excellent agreement of Eqs. (9)-(11) with the experimental results.

Application of the fitting procedure above to the experimental data in Fig. 1 led to the following estimates for the coefficient of free thermal diffusion $D_{0}=0.16 \mu \mathrm{m}^{2} / \mathrm{s}$, and for the potential amplitudes $\Delta U$ in (6) $\Delta U=9 k_{\mathrm{B}} T, 16 k_{\mathrm{B}} T$, and $23 k_{\mathrm{B}} T$.

With respect to our fit values, we note that, while the barrier height changes by about a factor of 3 for different laser intensities, the diffusion coefficient remains the same for all laser intensities. It is known from hydrodynamics at low Reynolds numbers [9] that the diffusion coefficient close to a wall becomes greatly reduced at distances not much larger than the particle radius $b$. In our case, the particle-wall distance is determined by the balance of the screened electrostatic repulsion between the particle and the wall on the one hand and, on the other hand, the sum of gravitational force (corrected with respect to buoyancy) and the force due to light pressure. Because the particle-wall repulsion is an exponential function of the distance [18], we expect the latter to change logarithmically weakly with laser intensity, resulting in practically the same $D_{0}$ for all three values used experimentally.

Because of the effect of the wall, the coefficient of free diffusion is smaller than the value given by the Stokes formula

$$
D_{0}<D_{\text {Stokes }}=k_{\mathrm{B}} T /(6 \pi \eta b),
$$

where the viscosity of water, $\eta$, is known to decrease with temperature [19]. Combining the result for $\eta(T)$ from Ref.
[19] with the fit value for $D_{0}$, we find from this inequality that the temperature in the region of the laser traps must be somewhat larger than $24^{\circ} \mathrm{C}$.

Given the estimates for the free diffusion coefficient and barrier height, the theoretical expressions (9)-(11) for the velocity $v$ and the diffusion coefficient $D$ are uniquely fixed for all laser intensities and rotation frequencies $\Omega$ considered experimentally in Fig. 1. Even though in the above procedure we had two fitting parameters at our disposition, the resulting excellent agreement with the experimental results is not at all self-evident. For a more detailed discussion of the physics behind the resonancelike diffusion enhancement observed in Fig. 1(b), we refer to [3-6].

\section{CONCLUSIONS}

The diffusion of a colloidal particle in a rotating array of laser traps can be modeled as a one-dimensional Brownian motion in a tilted periodic potential. We find excellent agreement between the theoretical curves for the velocity and the diffusion coefficient vs rotation frequency with the experimental results. While enhanced diffusion has been observed in previous experiments $[7,8]$, here we provide quantitative theoretical explanation of the experimental results.

The fit procedure introduced in this paper allows one to extract the important parameters characterizing the system, namely, the corrugation depth of the potential created by the laser traps and the coefficient of free thermal diffusion of the particle, as well as the local temperature of the particle's surroundings.

\section{ACKNOWLEDGMENTS}

We are grateful to the Deutsche Forschungsgemeinschaft (Grants No. RE 1344/3-1 and No. BE 1788/4-1, and the SFB 613) for financial support of this work.
[1] A. Einstein, Ann. Phys. 17, 549 (1905).

[2] S. Lifson and J. L. Jackson, J. Chem. Phys. 36, 2410 (1962).

[3] P. Reimann, C. Van den Broeck, H. Linke, P. Hänggi, J. M. Rubí, and A. Perez-Madrid, Phys. Rev. Lett. 87, 010602 (2001).

[4] P. Reimann, C. Van den Broeck, H. Linke, P. Hänggi, J. M. Rubí, and A. Perez-Madrid, Phys. Rev. E 65, 031104 (2002).

[5] D. Reguera, G. Schmid, P. S. Burada, J. M. Rubí, P. Reimann, and P. Hänggi, Phys. Rev. Lett. 96, 130603 (2006).

[6] G. Costantini and F. Marchesoni, Europhys. Lett. 48, 491 (1999).

[7] S.-H. Lee and D. G. Grier, Phys. Rev. Lett. 96, 190601 (2006).

[8] V. Blickle, T. Speck, U. Seifert, and C. Bechinger, Phys. Rev. E 75, 060101(R) (2007).

[9] J. Happel and H. W. Brenner, Low Reynolds Number Hydrodynamics (Prentice-Hall, Englewood Cliffs, NJ, 1965).

[10] B. Lin, J. Yu, and S. A. Rice, Phys. Rev. E 62, 3909 (2000).

[11] A. Ambari, B. Gauthier-Manuel, and E. Guyon, Phys. Fluids 28, 1559 (1985).
[12] K. Nelissen, V. R. Misko, and F. M. Peeters, Europhys. Lett. 80, 56004 (2007).

[13] E. Heinsalu, M. Patriarca, and F. Marchesoni, Phys. Rev. E 77, 021129 (2008).

[14] D. Babic and C. Bechinger, Phys. Rev. Lett. 94, 148303 (2005); D. Babic, C. Schmitt, I. Poberaj, and C. Bechinger, Europhys. Lett. 67, 158 (2004).

[15] L. P. Faucheux, G. Stolovitzky, and A. Libchaber, Phys. Rev. E 51, 5239 (1995).

[16] In the laboratory frame of reference, the velocity is given by $v-\Omega R$, while the diffusion coefficient remains unchanged, as can be inferred from (1) and (4).

[17] H. Risken, The Fokker-Planck Equation (Springer, Berlin, 1989).

[18] T. M. Squires and M. P. Brenner, Phys. Rev. Lett. 85, 4976 (2000).

[19] D. A. Berstad, B. Knapstad, M. Lamvik, P. A. Skjølsvik, K. Tørklep, and H. A. Øye, Physica A 151, 246 (1988). 